\title{
A delayed SEIQR epidemic model of COVID-19 in Tokyo area
}

\author{
Kazuo Maki* \\ MediEco R \& D Corporation \\ Hieidaira, Ohtsu 520-0016, Japan
}

*To whom correspondence should be addressed; E-mail: flute@music.email.ne.jp

The infection of COVID-19 has caused a global pandemic. In order to avoid excessive restriction to the social activity, a good strategy of quarantine based on a realistic model is expected. Several epidemic models with a quarantine compartment such as susceptible-exposed-infectious-quarantined-recovered (SEIQR) model have been applied. However, in the actual situation, the infection test and quarantine is often delayed from the beginning of the infectious stage.

This article presents a delayed SEIQR model to analyze the effect of the delay of quarantine. The latency period (compartment E) and the incubation period were assumed to be 3 days and 5 days, respectively. Considering that the presymptomatic infection ratio is 0.4 , the natural decay rate of the number of infectious patients was assumed to be 0.25 days $^{-1}$. The recovery rate was assumed to be 0.07 days $^{-1}$ from the typical PCR test positive period. The PCR test positive number in the period from March 10 to July 18 in 2020 in Tokyo area was analyzed. The delay time distribution of the quarantine was derived from the record of the symptom onset date, and was utilized to determine the delay time profile of quarantine in the model.

It was found that the major contributor to the infection control was the restraint of social contact. However, the quarantine action also contributed to reducing the reproduction number by the ratio of 0.88 and 0.8 in the period from March 10 to June 3 and from June 4 to July 18, respectively. The delay of quarantine was found to be well correlated to the effectiveness of quarantine. Therefore, a record on a symptom onset date is very important to estimate the effect of quarantine measure. The basic reproduction number was estimated to be 2.56. In view of the presymptomatic infection ratio 0.4 , it would be very hard to restrain the expansion of infection only by quarantining the symptomatic patients. 
medRxiv preprint doi: https://doi.org/10.1101/2020.08.18.20177709; this version posted November 7, 2020. The copyright holder for this preprint (which was not certified by peer review) is the author/funder, who has granted medRxiv a license to display the preprint in It is made available under a CC-BY-NC-ND 4.0 International license

\section{Introduction}

The novel coronavirus disease (COVID-19) has caused the infection expansion all over the world in the second quarter of 2020. A deterministic mathematical modeling of the outbreak of infection is expected to be a useful tool to analyze the situation and to suggest a plausible measure. In the SIR model [1], the total population $N$ of the area under consideration is divided into three compartments: susceptible $(S)$, infectious $(I)$, and recovered $(R)$ individuals. In the SEIR model [2], another compartment, exposed individuals $(E)$, is added to take a latency period into account. However, COVID-19 is different from most conventional infection cases in the point that unique symptom is not well established yet and that asymptomatic patients may be infectious [3],[4]. Since an infectious patient cannot be identified clearly, we have to restrain contact between the others in daily life. Influence to social activities is enormous. It is necessary to find and quarantine an infectious patient by some kind of test, and this quarantine measure deeply affects the situation of the infection expansion.

Models with a compartment for quarantined individuals are called SIQR model [5] or SEIQR model [6],[7],[8]. The occurrence of the transition from the component I to $\mathrm{Q}$, which is observed as a number of newly found infected patients, is usually assumed to be proportional to the number of infectious patients at large, and this proportionality coefficient is treated as a parameter called "quarantine rate". The outbreak of COVID-19 has been analyzed by many authors in terms of quarantine [9],[10],[11],[12],[13],[14]. The quarantine rate was estimated from available data under some simple assumptions or using statistical method [10] or AI model [11]. However, infected patients usually have a PCR examination after developing a symptom. Moreover when a check system is not well coordinated, they have to stand by for several days. Young et al. developed a delayed SEIQR model [15] including this delay effect, which was applied to COVID-19 by Vysarayani and Chatterjee [16]. In their model, the patient that has passed an assumed period is quarantined with an assumed probability, but if not quarantined, a chance of being quarantined is not left any longer. On the other hand, Utamura et al. [17] developed a model in that all the infectious patients are quarantined after passing an assumed period (14 days).

In this report, I will present a new model with two compartments of infectious patients: a quarantine waiting compartment and a quarantine possible compartment. Patients are quarantined with a quarantine rate only in the latter compartment. The quarantine rate defined in this model is a natural extension of that of non-delayed SIQR or SEIQR models. 
medRxiv preprint doi: https://doi.org/10.1101/2020.08.18.20177709; this version posted November 7, 2020. The copyright holder for this preprint (which was not certified by peer review) is the author/funder, who has granted medRxiv a license to display the preprint in It is made available under a CC-BY-NC-ND 4.0 International license

In the next section, a delayed SEIQR model will be formulated. In Section 3, the data of the daily found PCR positive patients in Tokyo area [18] will be analyzed. The last section will be devoted to a discussion and conclusion.

\section{A delayed SEIQR model}

In this section, a delayed SEIQR model will be formulated. As a compartment model, this model is similar to the delayed SEIQR model proposed by Young et al. [15]. As Young's model, I assumed that a patient stays in the compartment E for a definite period $\varepsilon$. As for the compartment I, which is the point of difference to Young's model, this compartment was divided into $\mathrm{J}$ and $\mathrm{K}$, and only those belonging to $\mathrm{K}$ were assumed to be quarantined. The patient stays in the compartment $\mathbf{J}$ for a period $\tau$ and automatically moves to the compartment $\mathbf{K}$. Here, a quarantine rate $q$ was introduced as an extension of that of non-delayed SIQR or SEIQR model. All the members in $\mathrm{K}$ were assumed to be continuously quarantined with a rate $q$ if not recovered. The parameter $\tau$ means a delay of the start of quarantine. If we expand the test to check earlier stage patient, $\tau$ can be decreased. In the case of non-delayed models $(\tau=0)$, a constant value is imposed on $q$ for the whole stage of an infectious patient at large. The flow chart is shown in Fig.1.

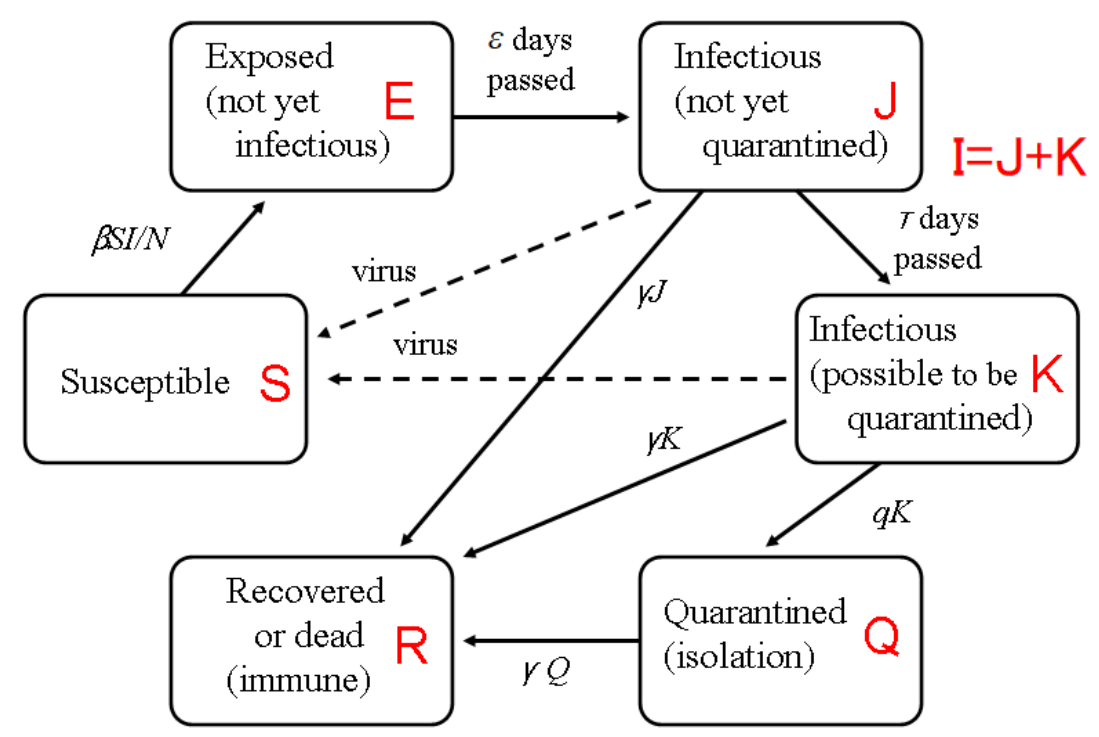

Figure 1: Flow chart of a delayed SEIQR model.

Thus, the delay differential equations are given as follows. (The time dependence of coefficients $\beta$ and $q$ is not indicated for simplicity. )

$$
\frac{d S(t)}{d t}=-\beta \frac{S(t) I(t)}{N}
$$




$$
\begin{aligned}
& \frac{d E(t)}{d t}=\beta \frac{S(t) I(t)}{N}-\beta \frac{S(t-\varepsilon) I(t-\varepsilon)}{N} \\
& \frac{d J(t)}{d t}=\beta \frac{S(t-\varepsilon) I(t-\varepsilon)}{N}-\beta \frac{S(t-\varepsilon-\tau) I(t-\varepsilon-\tau)}{N} e^{-\gamma \tau}-\gamma J(t) \\
& \frac{d K(t)}{d t}=\beta \frac{S(t-\varepsilon-\tau) I(t-\varepsilon-\tau)}{N} e^{-\gamma \tau}-q K(t)-\gamma K(t) \\
& \frac{d Q(t)}{d t}=q K(t)-\gamma Q(t) \\
& \frac{d R(t)}{d t}=\gamma I(t)+\gamma Q(t)
\end{aligned}
$$

, where

$$
\begin{aligned}
& I(t)=J(t)+K(t) \\
& N=S(t)+E(t)+J(t)+K(t)+R(t)
\end{aligned}
$$

The parameters $\beta, \gamma, q, \varepsilon$, and $\tau$ represent the transmission rate, the natural decay rate of the number of infectious patients, the quarantine rate, the latency period, and the delay of the start of quarantine, respectively. The transmission rate may be interpreted as the frequency of daily contact with others multiplied by the transmission risk from an encountered infectious patient. Birth and death processes are neglected here. The factor $\exp (-\gamma \tau)$ in Eqs. (2.3) and (2.4) is necessary to take into account the decay with the rate $\gamma$ in the compartment $\mathbf{J}$.

It should be noted here that the PCR test positive result does not always mean infectiousness because PCR test does not detect active virus but detects only RNA [19],[20]. The recovery rate, denoted as $\gamma^{\prime}$, is defined here as the inverse of the averaged PCR test positive period. This rate is smaller than the natural decay rate $\gamma$ of the number of infectious patients. Thus we have to assume similar compartments $\mathrm{J}^{\prime}$ and $\mathrm{K}$ ' for PCR test positive patients as an extension of $\mathbf{J}$ and $\mathrm{K}$, respectively. The number of PCR test positive patients in the compartment K', denoted as $K^{\prime}(t)$, satisfies the following additional delay differential equation.

$$
\frac{d K^{\prime}(t)}{d t}=\beta \frac{S(t-\varepsilon-\tau) I(t-\varepsilon-\tau)}{N} e^{-\gamma^{\prime} \tau}-q K^{\prime}(t)-\gamma^{\prime} K^{\prime}(t)
$$

Then the number of the newly found PCR test positive patient is

$$
\Delta Q(t)=q K^{\prime}(t)
$$

When the parameters are made $\varepsilon=0, \tau=0, \varepsilon=\tau=0, \tau=q=0$, and $\varepsilon=\tau=q=0$, this model becomes a delayed SIQR model, an SEIQR model, an SIQR model, an SEIR model, and an SIR model respectively. 
medRxiv preprint doi: https://doi.org/10.1101/2020.08.18.20177709; this version posted November 7, 2020. The copyright holder for this preprint (which was not certified by peer review) is the author/funder, who has granted medRxiv a license to display the preprint in It is made available under a CC-BY-NC-ND 4.0 International license.

Hereafter, I will consider only the early stage of outbreak. I made an approximation as $S=N$, and only Eqs. (2.3), (2.4), (2.7), (2.9), and (2.10) will be used. These equations are rewritten as

$$
\begin{aligned}
& \frac{d I(t)}{d t}=\beta I(t-\varepsilon)-q K(t)-\gamma I(t) \\
& \frac{d K(t)}{d t}=\beta I(t-\tau-\varepsilon) e^{-\gamma \tau}-q K(t)-\gamma K(t) \\
& \frac{d K^{\prime}(t)}{d t}=\beta I(t-\tau-\varepsilon) e^{-\gamma^{\prime} \tau}-q K^{\prime}(t)-\gamma^{\prime} K^{\prime}(t)
\end{aligned}
$$

Next, I will establish the relation of the model parameters to the actual epidemic data. In addition to the PCR test positive fixation date $T_{\mathrm{f}}$, the epidemic data usually contain the symptom onset date $T_{\mathrm{s}}$ if the patient is symptomatic. The delay of quarantine is defined as $T_{\mathrm{d}}=T_{\mathrm{f}}-T_{\mathrm{s}}+T_{\mathrm{p}}$, where $T_{\mathrm{p}}$ is the presymptomatic infectious period. (I will assume later that $T_{\mathrm{p}}=2$ days.) In order to derive the expression of the distribution function of $T_{\mathrm{d}}$, we have to calculate the probability $P(t)$ that a patient who became infectious at $t=0$ is not quarantined while keeping PCR test positive. Since the decay rate is $\gamma^{\prime}$ before the start of quarantine $(t<\tau)$, and then $\gamma^{\prime}+q$ if $t>\tau$.

$$
\begin{array}{ll}
P(t)=e^{-\gamma^{\prime} t} & \text { if } t<\tau, \\
P(t)=e^{-\gamma^{\prime} \tau} e^{-\left(\gamma^{\prime}+q\right)(t-\tau)} & \text { if } t \geq \tau
\end{array}
$$

The probability of daily quarantine in the PCR test is given by $q P(t)(t \geq \tau)$, which is rewritten as

$$
q P(t)=p_{q} \varphi(t)
$$

where $\varphi(t)=0 \quad$ if $t<\tau$,

$$
\begin{aligned}
& \varphi(t)=\left(\gamma^{\prime}+q\right) e^{-\left(\gamma^{\prime}+q\right)(t-\tau)} \text { if } t \geq \tau \\
& p_{q}=\frac{q e^{-\gamma^{\prime} \tau}}{\left(\gamma^{\prime}+q\right)}
\end{aligned}
$$

The function $\varphi(t)$ and the coefficient $p_{\mathrm{q}}$ are interpreted as the normalized distribution of the delay time of quarantine and the ratio of the PCR test detection of infected patient, respectively. It should be noted that $\varphi(t)$ can be observed, but $p_{\mathrm{q}}$ cannot be observed directly. Although the observed quarantine delay distribution is different from the distribution $\varphi(t)$ of the model, the most 
medRxiv preprint doi: https://doi.org/10.1101/2020.08.18.20177709; this version posted November 7, 2020. The copyright holder for this preprint (which was not certified by peer review) is the author/funder, who has granted medRxiv a license to display the preprint in It is made available under a CC-BY-NC-ND 4.0 International license .

important parameter is the averaged delay time $\left\langle T_{\mathrm{d}}\right\rangle$. Hereafter, $\langle>$ denotes an average by the observed time distribution of the delay of quarantine. Model parameters should give the same averaged delay time.

$$
<T_{d}>=\tau+\frac{1}{\left(\gamma^{\prime}+q\right)}
$$

If we assume that the standard deviation $\sigma$ should be the same as observed,

$$
\sigma=\frac{1}{\left(\gamma^{\prime}+q\right)}
$$

These equations determine $\tau$ and $q$.

If we replace $\gamma^{\prime}$ with $\gamma, P(t)$ will be the probability that an infected patient is not quarantined while maintaining infectiousness. The effective reproduction number $R_{e}$ is given by carrying out the time integration of $P(t)$ (Eq.(2.14) where $\gamma^{\prime}$ is replaced with $\gamma$ ) multiplied by $\beta$.

$$
R_{e}=\frac{\beta}{\gamma}\left\{1-\frac{q e^{-\gamma \tau}}{(\gamma+q)}\right\}
$$

The second term in the parenthesis \{\} means the ratio of the infectious patient detected and quarantined by the PCR test. The ratio $R_{e} /(\beta / \gamma)$ may be interpreted as the effect of quarantine when excluding the social contact avoidance effect. This ratio is expressed in terms of the characteristic parameters of the distribution of the delay of quarantine.

$$
R_{e}=\frac{\beta}{\gamma}\left\{1-\frac{\left(\frac{1}{\sigma}-\gamma^{\prime}\right)}{\left(\frac{1}{\sigma}+\gamma-\gamma^{\prime}\right)} e^{-\gamma\left(<T_{d}>-\sigma\right)}\right\}
$$

As an example, the ratio $R_{e} /(\beta / \gamma)$ is plotted in Fig. 2 , using the values of $\gamma=0.25$ days $^{-1}$ and $\gamma^{\prime}=0.25$ days $^{-1}$ as will be determined in the next section. It is well correlated with the averaged delay of quarantine. In Appendix $\mathrm{A}, R_{e}$ is expressed directly by an observed time distribution of the delay of quarantine in a different epidemic model.

$$
R_{e}=\frac{\beta}{\gamma}\left(1-\frac{<e^{\left(\gamma^{\prime}-\gamma\right) T_{d}}>}{<e^{\gamma^{\prime} T_{d}}>}\right)
$$

The accuracy of the approximation in Eq. (2.21) can be evaluated by this equation.

In the case of non-delayed models $(\tau=0)$ the reproduction number is expressed 
medRxiv preprint doi: https://doi.org/10.1101/2020.08.18.20177709; this version posted November 7, 2020. The copyright holder for this preprint (which was not certified by peer review) is the author/funder, who has granted medRxiv a license to display the preprint in It is made available under a CC-BY-NC-ND 4.0 International license .

as $R_{e}=\beta /(\gamma+q)$. This case is indicated by small triangles in Fig.2. The basic reproduction number is estimated as $R_{0}=\beta / \gamma$ using the earliest value in $\beta$.

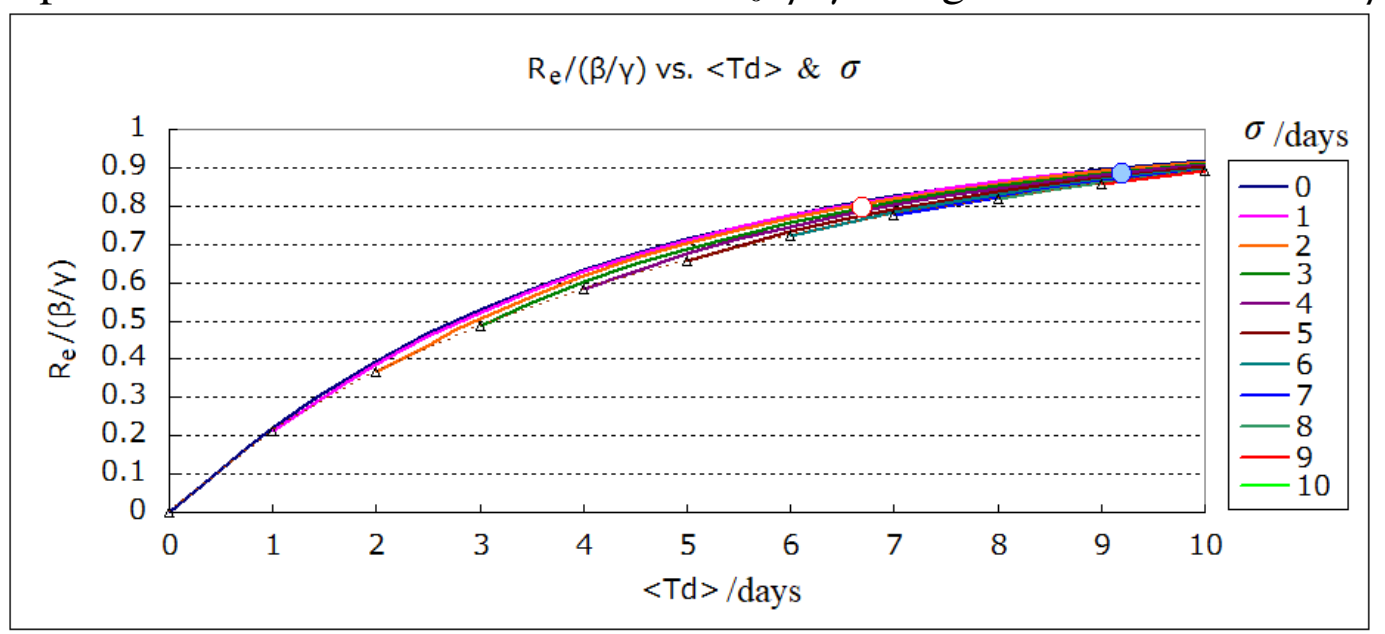

Figure 2. Effect of quarantine on the reproduction number

$\left\langle T_{\mathrm{d}}\right\rangle$ and $\sigma$ denote an average and a standard deviation of the delay of quarantine, respectively. The small triangles correspond to the case that $\left\langle T_{\mathrm{d}}\right\rangle=\sigma$ or $\tau=0$. Two circles correspond to the Tokyo area data in Sec.3.

There is a stationary solution proportional to $\exp (\lambda t)$ in Eqs. (2.11) and (2.12). The coefficient $\lambda$ is called the infection expansion coefficient, and it satisfies the following relation, which will be useful not only in finding $\beta$ from the observed time profile of infection but also in predicting the expansion rate $\lambda$.

$$
\beta e^{-\lambda \varepsilon}=\lambda+\gamma+\frac{q(\lambda+\gamma) e^{-(\lambda+\gamma) \tau}}{\lambda+\gamma+q\left(1-e^{-(\lambda+\gamma) \tau}\right)}
$$

The left hand side may be interpreted as an effective transmission rate, which depends on the latency period $\varepsilon$. If the infection is expanding $(\lambda>0)$ or shrinking $(\lambda<0)$, the effective transmission rate is reduced or enhanced, respectively.

\section{Analysis of the time profile of infection in Tokyo area}

In this section, I will analyze the data of the number of PCR test positive person who were living and tested in Tokyo area (Tokyo, Kanagawa, Saitama, and Chiba) [18]. The test period is from March 10 to July 18 in 2020. The total number of patients is 13,978 . The parameters $\gamma, \gamma^{\prime}$, and $\varepsilon$ will be estimated from references, and the parameters $\tau$ and $q$ will be derived from the observed quarantine delay distribution. Finally, the parameter $\beta$ will be chosen so as to reproduce the time profile of the number of PCR test positive patients.

The incubation period has a wide distribution and the average is about 5 days [21]. It is also known that patients are infectious 2 days before the onset of symptom [3],[4]. Thus, I assumed that the latency period $\varepsilon$ is 3 days. I assumed 
medRxiv preprint doi: https://doi.org/10.1101/2020.08.18.20177709; this version posted November 7, 2020. The copyright holder for this preprint (which was not certified by peer review) is the author/funder, who has granted medRxiv a license to display the preprint in It is made available under a CC-BY-NC-ND 4.0 International license .

that the natural decay rate $\gamma$ of the number of infectious patients is 0.25 days $^{-1}$ so as to make the ratio of presymptomatic infection 0.4 as observed [3],[4]. Since the PCR test positive period has a wide distribution [22],[23], I assumed 14 days as a typical period. The recovery rate $\gamma^{\prime}$ was set to be $1 / 14=0.07$ days ${ }^{-1}$. The time profile of probability of being infectious is compared with that of being PCR test positive in Fig.3.

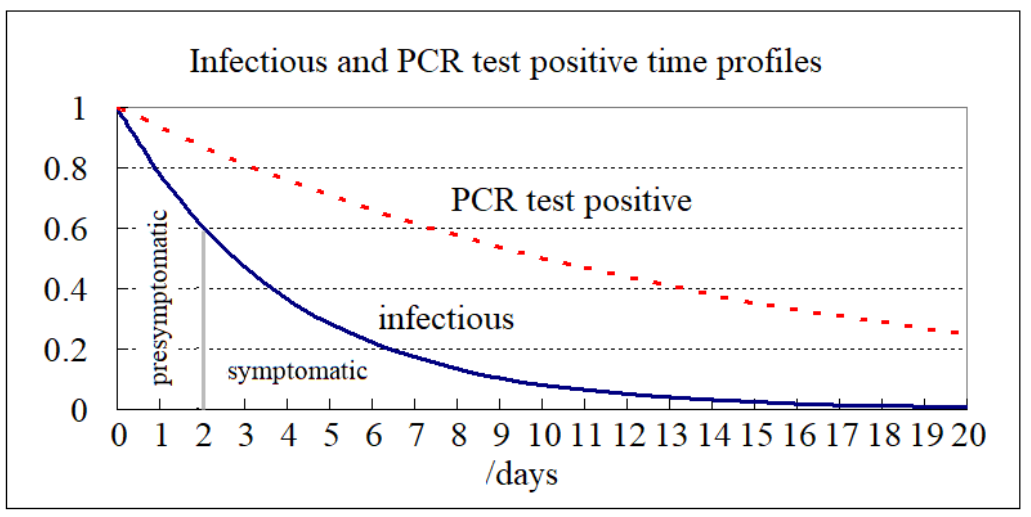

Figure 3. Comparison of infectious and PCR test positive time profiles

The symptom onset date $\left(T_{\mathrm{s}}\right)$ was recorded in $24 \%$ of the data of Tokyo area [18]. The daily average of the delay of quarantine $\left(T_{\mathrm{d}}=T_{\mathrm{f}}-T_{\mathrm{s}}+T_{\mathrm{p}}\right)$ is shown in Fig.4. Although the data are scattered, the delay in June and July is apparently shorter than that in March, April, and May. I divided the whole period at the point between June 3 and 4.

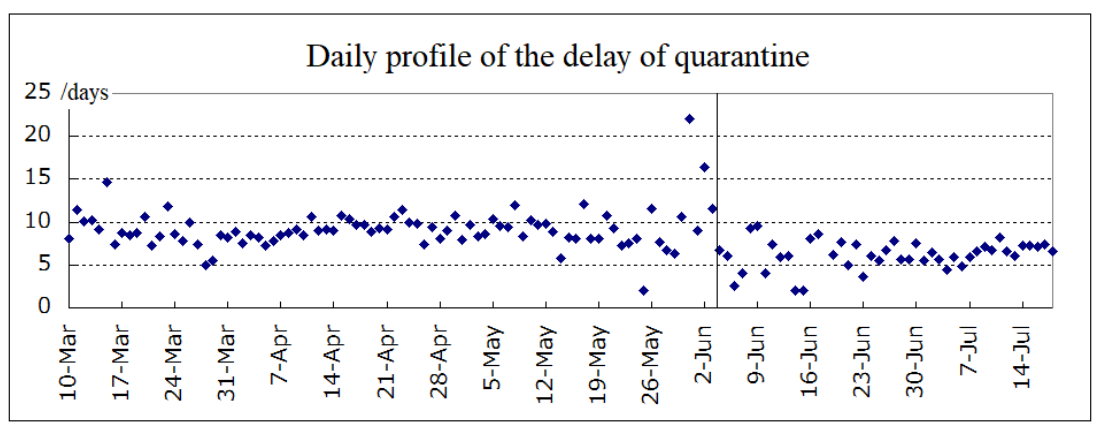

Figure 4. Daily profile of the delay of quarantine

The delay of the quarantine is defined as the period from the start of infectiousness to the PCR test positive fixation day. It is assumed here that the start of infectiousness is 2 days earlier than the onset of symptom.

The distributions of the delay of quarantine in these periods are shown in Fig.5. The average $\left(\left\langle T_{\mathrm{d}}\right\rangle\right)$ and the standard deviation $(\sigma)$ of the delay is 9.17 days and 4.24 days respectively in the former period, and 6.69 days and 3.08 days respectively in the latter period. The parameters $\tau, q$, and the effect of quarantine $\left(R_{e} /(\beta / \gamma)\right)$ were calculated by Eqs. (2.18), (2.19), and (2.20). The result was that $\tau=4.93$ days, $q=0.166$ days $^{-1}$, and $R_{e} /(\beta / \gamma)=0.884$ in the former period, while 
medRxiv preprint doi: https://doi.org/10.1101/2020.08.18.20177709; this version posted November 7, 2020. The copyright holder for this preprint (which was not certified by peer review) is the author/funder, who has granted medRxiv a license to display the preprint in It is made available under a CC-BY-NC-ND 4.0 International license .

$\tau=3.6$ days and $q=0.25$ days $^{-1}$, and $R_{e} /(\beta / \gamma)=0.795$ in the latter period. These are indicated by two small circles in Fig.2. (In the case of the exact expression (Eq. (2.22)), $R_{e} /(\beta / \gamma)=0.879$ and $R_{e} /(\beta / \gamma)=0.792$ in the former and the latter period, respectively. This means that the present model is a good approximation.) Since a small change of parameters does not affect the result much as far as the effect of quarantine is the same, I applied a common value of $\tau=4$ days for a convenience on the numerical calculation, and $q$ was readjusted so as to keep the effect of quarantine. I obtained the values as $q=0.116$ and $q=0.314$ for the former and the latter period, respectively. If we set that $\tau=0$ : i.e. in the case of nondelayed model, $q=0.039$ days $^{-1}$ and $R_{e} /(\beta / \gamma)=0.865$ in the former period, $q=0.0795$ and $R_{e} /(\beta / \gamma)=0.759$ in the latter period. The delay time profiles in the two cases ( $\tau=4$ and $\tau=0$ ) are compared with the observed profiles in Fig.5.

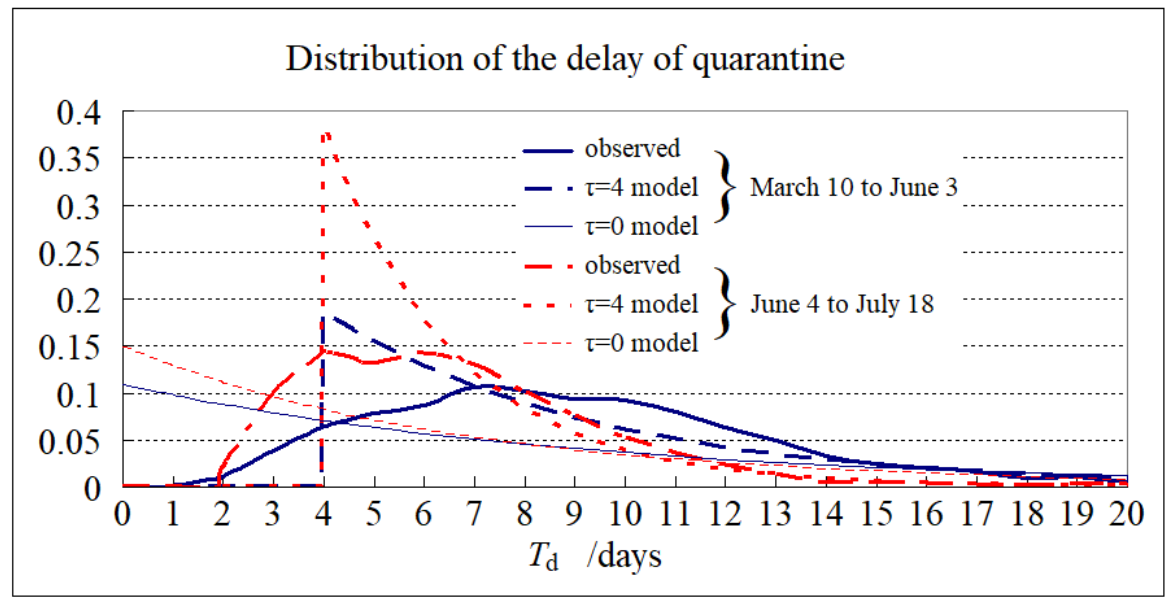

Figure 5. Distribution of the delay of quarantine

Numerical calculation was made by the fourth order Runge-Kutta method with time step $=0.1$ days. The PCR test positive person number data of Tokyo area $\Delta Q(t)$ in the period from March 10 to July 18 [18] was reproduced by choosing the parameter $\beta$. The time profile of infection is shown in Fig.6. The parameters $\beta$ and $q$ and the effective reproduction number $R_{\mathrm{e}}$ in each period are listed in Table 1. As for the parameters $\beta$ and $q$, the moving average of 7 days of the values in Table 1 was adopted as shown in Fig.6. The small protuberance in $\Delta Q(t)$ around June 3 reflects the change in the parameter $q$ (the enhancement of PCR test). The basic reproduction number $R_{0}$ is found to be $0.640 / 0.25=2.56$. 


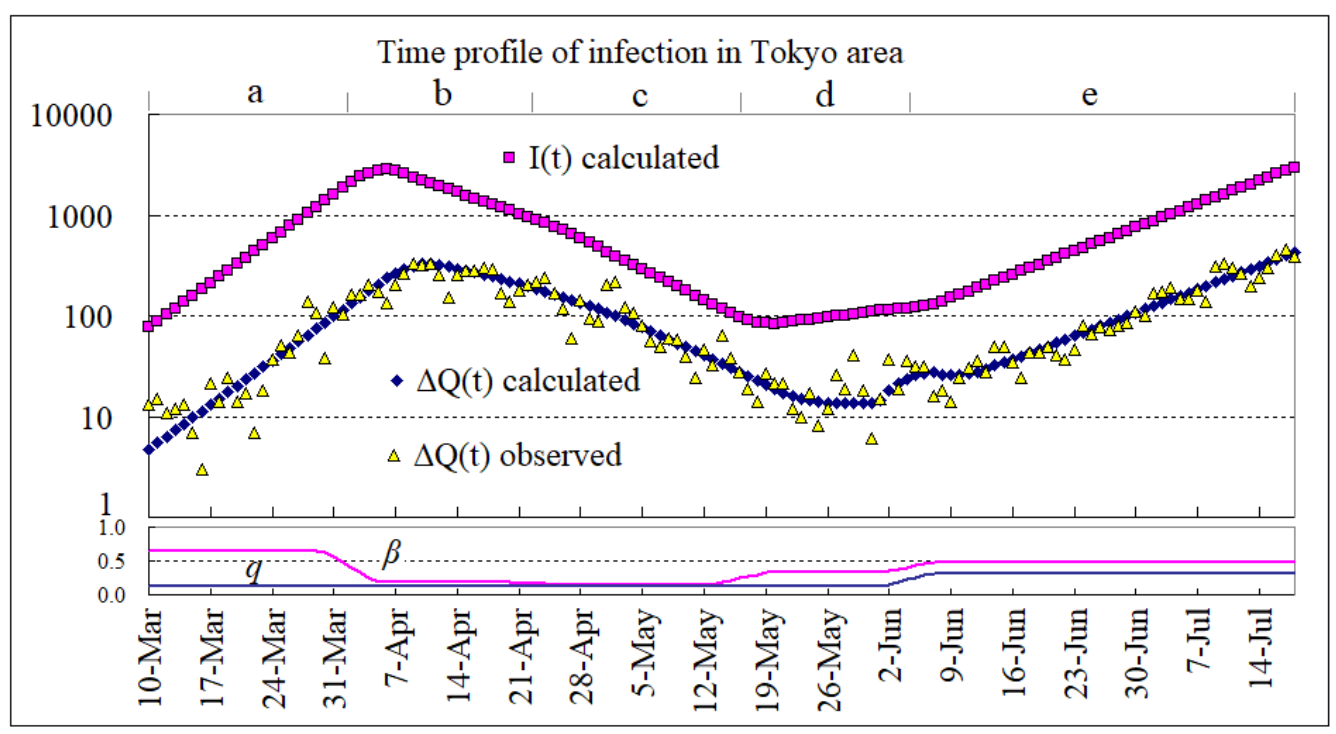

Figure 6: Time profile of infection in Tokyo area

Vertical axis is a common logarithm. The observed data are from public data [18]. $I(t)$ denotes the number of infectious patients not quarantined. $\Delta Q(t)$ denotes the number of daily PCR test positive patients. Symbols on the top represent five periods. Parameters $\beta$ and $q$ are shown underneath the main graph. The parameter $\tau$ is set to be 4 days.

Table 1: Parameter set and effective reproduction number

The parameter $\tau$ is set to be 4 days. The symbols $\beta, q$, and $R_{e}$ denote transmission rate (days ${ }^{-1}$ ), quarantine rate $\left(\right.$ days $\left.^{-1}\right)$, and the effective reproduction number, respectively. $\beta / 0.640$ or $R_{e} /(\beta / \gamma)$ means a contribution of $\beta$ or that of quarantine, respectively, to the reduction ratio of the reproduction number.

\begin{tabular}{|c|c|c|c|c|c|}
\hline Period & a: Mar 10-Apr 1 & b: Apr 2-Apr 22 & c: Apr 23-May 15 & d: May 16-Jun 3 & e: Jun 4-Jul 18 \\
\hline$\beta$ & 0.640 & 0.180 & 0.146 & 0.329 & 0.474 \\
\hline$q$ & 0.116 & 0.116 & 0.116 & 0.116 & 0.314 \\
\hline$R_{e}$ & 2.26 & 0.64 & 0.52 & 1.16 & 1.51 \\
\hline$\beta / 0.640$ & 1 & 0.28 & 0.23 & 0.51 & 0.74 \\
\hline$R_{e} /(\beta / \gamma)$ & 0.884 & 0.884 & 0.884 & 0.884 & 0.795 \\
\hline
\end{tabular}

The reduction ratio of the reproduction number is a product of the social action inhibiting effect $(\beta / 0.640)$ and the quarantine effect $\left(R_{e} /(\beta / \gamma)\right)$. They are shown in Table.1. The effect of the social behavior restraint is dominant on the whole. It is remarkable in the period b and $\mathrm{c}$ in particular. Death of a famous comedy actor was reported, and people checked social behavior. An emergency declaration was issued, and social contact restraint of $80 \%$ was recommended. After the emergency declaration was released, $\beta$ increased in the period $\mathrm{d}$ and $\mathrm{e}$, but the PCR check system has been strengthened by period e by the ratio $0.204 / 0.115=1.77$. This eased infection expansion. The time profile of infection after those periods is not shown here. The social behavior was limited again, and infection calmed down a little throughout August and September. However, in view of the economical influence, the quarantine action should be strengthened. 
medRxiv preprint doi: https://doi.org/10.1101/2020.08.18.20177709; this version posted November 7, 2020. The copyright holder for this preprint (which was not certified by peer review) is the author/funder, who has granted medRxiv a license to display the preprint in It is made available under a CC-BY-NC-ND 4.0 International license .

In order to grasp the situation, the curves which make the infection expansion rate $\lambda$ fixed (Eqs. (2.18), (2.19), and (2.23)) are drawn in the plane of $\beta$ and $\left\langle T_{\mathrm{d}}\right\rangle$ in Fig.7. (Note that $\lambda=-0.05$ or -0.1 means $30 \%$ or $50 \%$ decrease of infection a week, respectively.) Since there is a small dependence on $\sigma$, the two extreme cases are drawn. One is the case that $q=\infty\left(\sigma=0\right.$; i.e. $\left.\left\langle T_{\mathrm{d}}\right\rangle=\tau\right)$, the other is the case that $\tau=0 \quad\left(\left\langle T_{\mathrm{d}}\right\rangle=\sigma\right.$ : i.e. non-delayed SEIQR model). The result of the analysis in Tokyo area is indicated by the grey dots. As an example, let us start from the period e. If the quarantine measure is the same, we have to restrain the social activity by $35 \%$ to get over the $\lambda=0$ line $\left(\beta=0.31\right.$ day $\left.^{-1}\right)$, but if the quarantine measure could be strengthened as that $\left\langle T_{\mathrm{d}}\right\rangle=4$ days, only $16 \%$ reduction of $\beta$ would be sufficient to get over the $\lambda=0$ line $\left(\beta=0.4\right.$ day $\left.^{-1}\right)$. The economic impact would be much mitigated. However, when only the symptomatic patient is quarantined, $\left\langle T_{\mathrm{d}}\right\rangle$ cannot be made shorter than 2 days. This also means that $R_{\mathrm{e}}$ cannot be made less than $1.04=0.4 R_{0}$, if we recover the level of social activity before the outbreak of COVID-19. (The number 0.4 is the presymptomatic ratio of infection.) Thus the quarantine of presymptomatic patient is necessary.

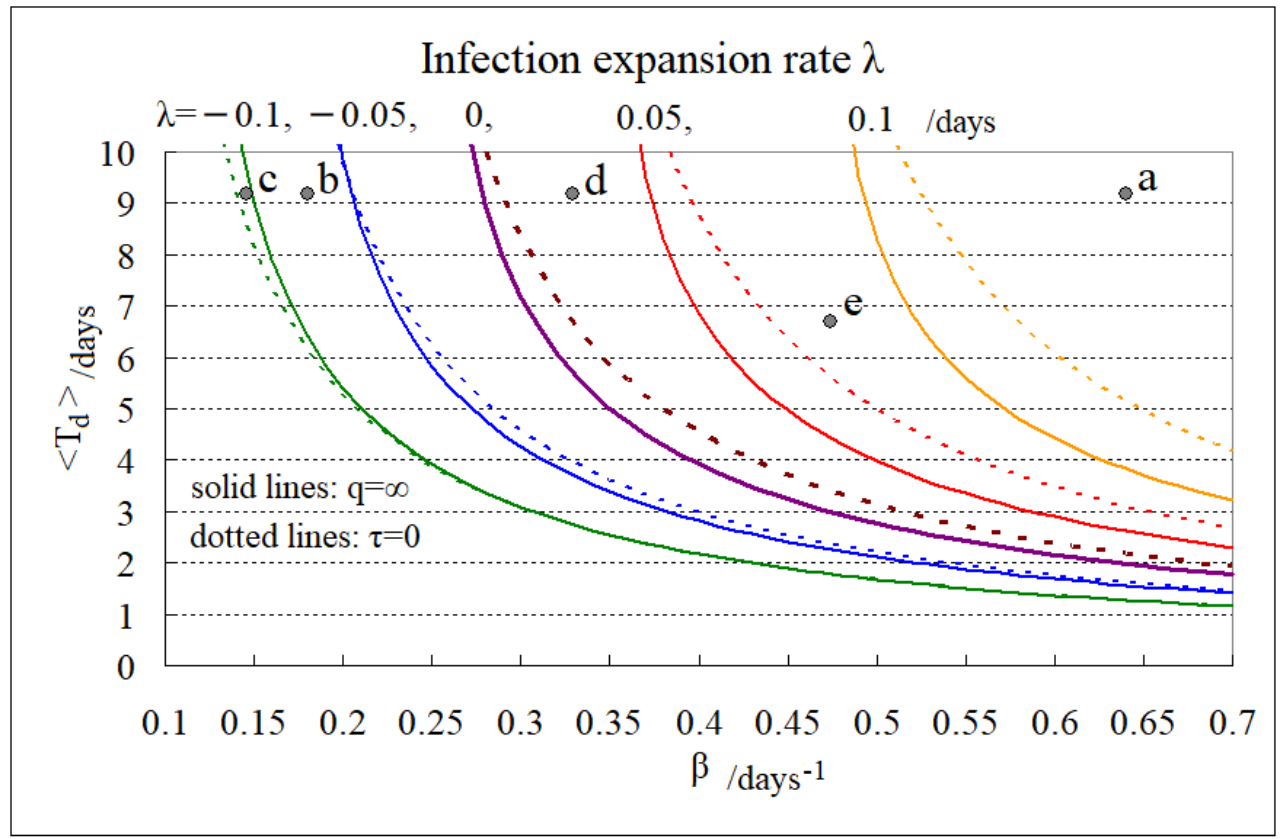

Figure 7. Infection expansion rate

The curves which make infection expansion rate $\lambda=-0.1,-0.05,0,0.05,0.1$ are depicted in two cases. One is the case that $q=\infty(\sigma=0)$, the other is the case that $\tau=0\left(\left\langle T_{\mathrm{d}}\right\rangle=\sigma\right)$.

\section{Summary and discussion}

Conventional SIQR or SEIQR (non-delayed) models assume that the number of quarantined patients daily is proportional to the number of infectious patients 
medRxiv preprint doi: https://doi.org/10.1101/2020.08.18.20177709; this version posted November 7, 2020. The copyright holder for this preprint (which was not certified by peer review) is the author/funder, who has granted medRxiv a license to display the preprint in It is made available under a CC-BY-NC-ND 4.0 International license .

at large. As shown by thin lines in Fig.5, this supposition is equivalent to assuming an exponential distribution of the quarantine delay from the beginning of infectiousness. However, the observed delay time distribution looks very different from that assumed in these models. In order to reflect the essential characteristics of the distribution, I expanded conventional SEIQR models with a new parameter $\tau$ describing the delay of the start of quarantine. I also estimated the natural decay rate $\gamma$ of the number of infectious patients from the observed ratio of presymptomatic infection. The decay rate was found to be much larger than usual estimates from medical recovery data. Then I introduced a recovery rate $\gamma^{\prime}$ as the inverse of a PCR test positive period to reproduce the observed PCR test positive number.

I found that the quarantine effect on the reduction of the effective reproduction number is closely related to the averaged delay of quarantine as is shown in Fig.2. This means that we can estimate the effect of quarantine measure directly. (The exact relation is given in Eq. (2.22).) Although this estimation from the averaged delay is also possible in the non-delayed models, the estimation itself tends to be rather optimistic. It should also be noted that, in the non-delayed models, a prediction in terms only of $\beta$ and $q$ might become too optimistic because the difficulty of shortening the delay of quarantine is often underestimated.

The infection expansion or shrinking can be predicted in Fig.7, where the infection expansion rate $\lambda$ is expressed in terms of the transmission rate $\beta$ and the averaged delay of quarantine $\left\langle T_{\mathrm{d}}\right\rangle$. As a point of the infection measure, I share a view with $\mathrm{He}$ et al. [3] and Chun et al. [4]. The quarantine of presymptomatic patient is necessary to recover the normal social activities.

This report has the following limitations.

1. I did not distinguish asymptomatic patients from presymptomatic patients. The data of asymptomatic case are very limited [22],[23],[24]. If both of its proportion and infectiousness are not negligible, it is necessary to take into account the difficulty of checking the asymptomatic case. One of the methods was developed in Appendix A, where some portion of patients can be left unchecked.

2. I assumed that the time profile of the onset of transmission (infectiousness) is an exponential type distribution. Actually it is estimated that there is a peak around the symptom onset time [3],[4]. This might affect the result when the average of quarantine delay is as short as the period of presymptomatic infectionon (2 days).

3. The symptom onset date was recorded only in $24 \%$ of the data. This loss of 
data might have an influence on the result of the estimation of the quarantine effect. Especially, I am afraid that there may be a tendency of forgetting to record the symptom onset date in the case of presymptomatic patient found by contact tracing. There would be a possibility of underestimate of the quarantine effect if the quarantine measure is further strengthened in future. A correct record on a symptom onset date is important to evaluate the quarantine effect.

\section{Acknowledgments}

I would like to thank Drs. T. Odagaki and H. Mitsuishi for valuable discussion, and thank J.A.G Japan corporation for the epidemic data.

Appendix A: Exact evaluation of the quarantine effect and the effective reproduction number

In order to reproduce exactly the same quarantine delay distribution as the observed one, we have to construct a different epidemic model similar to Young's model [15]. The compartment of infectious patient I and that of the PCR test positive patient $\mathrm{I}^{\prime}$ are divided into many compartments $\mathrm{J}_{\tau}$ and $\mathrm{J}_{\tau}$, respectively. The suffix $\tau$ means that all the members in that compartment are quarantined when $\tau$ days passed (corresponding to the case that $\sigma=0$ in the main text). (I do not need the compartment $\mathrm{K}$ nor $\mathrm{K}^{\prime}$.) In this report, I discarded the difficulty of checking asymptomatic patients. If we take that difficulty into account, we have to include a compartment with $\tau=\infty$. Equations $(2.3),(2.4),(2,7)$ and (2.9) are replaced by the following equations.

$$
\begin{aligned}
& \frac{d J_{\tau}(t)}{d t}=p_{\tau} \beta \frac{S(t-\varepsilon) I(t-\varepsilon)}{N}-p_{\tau} \beta \frac{S(t-\varepsilon-\tau) I(t-\varepsilon-\tau)}{N} e^{-\gamma \tau}-\gamma J_{\tau}(t) \\
& \frac{d J_{\tau}^{\prime}(t)}{d t}=p_{\tau} \beta \frac{S(t-\varepsilon) I(t-\varepsilon)}{N}-p_{\tau} \beta \frac{S(t-\varepsilon-\tau) I(t-\varepsilon-\tau)}{N} e^{-\gamma^{\prime} \tau}-\gamma^{\prime} J_{\tau}^{\prime}(t) \\
& I(t)=\sum_{\tau} J_{\tau}(t)
\end{aligned}
$$

, where $J_{\tau}$ denotes the number of the infectious patients and $J_{\tau}^{\prime}$ denotes that of the PCR test positive patients in the compartments $\mathrm{J}_{\tau}$ and $\mathrm{J}_{\tau}^{\prime}$, respectively. The parameter $p_{\tau}$ means the ratio of patients entering the compartment $\mathrm{J}_{\tau}$ and $\mathrm{J}_{\tau}{ }_{\tau}$. With an approximation that $S=N$, if a patient enters the compartment $\mathrm{J}_{\tau}$ and $\mathrm{J}_{\tau}{ }_{\tau}$ at $t=0$, the probability of being infectious without being quarantined is $\exp (-\gamma t)$, and that of being PCR test positive is $\exp \left(-\gamma^{\prime} t\right)$. Thus, the effective reproduction 
medRxiv preprint doi: https://doi.org/10.1101/2020.08.18.20177709; this version posted November 7, 2020. The copyright holder for this preprint (which was not certified by peer review) is the author/funder, who has granted medRxiv a license to display the preprint in

number is

$$
R_{e}=\frac{\beta}{\gamma}\left(1-\sum_{\tau} p_{\tau} e^{-\gamma \tau}\right)
$$

The time distribution of the number of quarantined PCR test positive patients is discrete.

$$
f_{\tau}=C p_{\tau} e^{-\gamma^{\prime} \tau}
$$

$C$ is a normalization constant. Since there is no observed data corresponding to $f_{\infty}, C$ should be determined as follows.

$$
\sum_{\tau}^{\prime} f_{\tau} e^{\gamma^{\prime} \tau}=C\left(1-p_{\infty}\right)
$$

, where $\Sigma_{\tau}^{\prime}$ means sum with respect of $\tau<\infty$.

$$
p_{\tau}=\frac{\left(1-p_{\infty}\right) f_{\tau} e^{\gamma^{\prime} \tau}}{\sum_{\tau}^{\prime} f_{\tau} e^{\gamma^{\prime} \tau}} \text { if } \tau \neq \infty
$$

The effective reproduction number is expressed in terms of the time distribution of the delay of quarantine.

$$
R_{e}=\frac{\beta}{\gamma}\left(1-\left(1-p_{\infty}\right) \frac{\sum_{\tau}^{\prime} f_{\tau} e^{\left(\gamma^{\prime}-\gamma\right) \tau}}{\sum_{\tau}^{\prime} f_{\tau} e^{\gamma^{\prime} \tau}}\right)
$$

Equation (2.22) is obtained by assuming that $p_{\infty}=0$.

When the expansion coefficient $\lambda$ is estimated from the time profile of the number of daily PCR test positive persons, the transmission coefficient $\beta$ can be calculated from the following equation corresponding to Eq. (2.23).

$$
\beta e^{-\lambda \varepsilon}=\frac{\lambda+\gamma}{\sum_{\tau} p_{\tau}\left(1-e^{-(\lambda+\gamma) \tau}\right)}
$$

Therefore, knowing the time profile of the number of daily PCR test positive persons and that of the delay of quarantine, and neglecting the influence of asymptomatic infectious patients $\left(p_{\infty}=0\right)$, we can derive directly the effective reproduction number using Eqs. (A.7) and the following equation.

$$
R_{e}=e^{\lambda \varepsilon} \frac{(\lambda+\gamma) \sum_{\tau} p_{\tau}\left(1-e^{-\gamma \tau}\right)}{\gamma \sum_{\tau} p_{\tau}\left(1-e^{-(\lambda+\gamma) \tau}\right)}
$$


medRxiv preprint doi: https://doi.org/10.1101/2020.08.18.20177709; this version posted November 7, 2020. The copyright holder for this preprint (which was not certified by peer review) is the author/funder, who has granted medRxiv a license to display the preprint in It is made available under a CC-BY-NC-ND 4.0 International license .

\section{References}

[1] W.O. Kermack and A.G. McKendrick, Contributions to the mathematical theory of epidemics-I, Proceedings of the Royal Society 115A, 700-721 (1927)

[2] R.M. Anderson and R.M. May: Directly transmitted infections diseases: control by vaccination, Science 26 Feb Vol.215, Issue 4536, 1053-1060 (1982)

[3] X. He et al., Temporal dynamics in viral shedding and transmissibility of COVID-19, Nat Med 26, 672-675 (2020), https://doi.org/10.1038/s41591020-0869-5

[4] J.Y. Chun et al., Transmission onset distribution of COVID-19, International Journal of Infectious Diseases 99 (2020) 403-407, 21 July 2020

[5] H. Hethcote, et al., Effects of quarantine in six endemic models for infectious diseases, Mathematical Biosciences 180, 141-160 (2002)

[6] D.J. Gerberry and F.A. Milner, An SEIQR model for childhood diseases, J Math Biol. 59(4), 535-561 (2009), doi:10.1007/s00285-008-0239-2

[7] P. Yongzhena et al., A delayed SEIQR epidemic model with pulse vaccination and the quarantine measure, Computers and Mathematics with Applications 58, 135-145 (2009)

[8] T. Li and Y. Xue, Global Stability Analysis of a Delayed SEIQR Epidemic Model with Quarantine and Latent, Applied Mathematics, 4, 109-117 (2013)

[9] L. Peng et al., Epidemic analysis of COVID-19 in China by dynamical modeling, doi: https://doi.org/10.1101/2020.02.16.20023465

[10] H-Y. Yuan et al., Effectiveness of quarantine measure on transmission dynamics of COVID-19 in Hong Kong, doi: https://doi.org/10.1101/2020.04.09.20059006

[11] R. Dandekar and G. Barbastathis, Quantifying the effect of quarantine control in Covid-19 infectious spread using machine learning, doi: https://doi.org/10.1101/2020.04.03.20052084

[12] M.S. Aronna et al., A model for COVID-19 with isolation, quarantine and testing as control measures, doi: https://doi.org/10.1101/2020.05.29.20116897

[13] T. Odagaki, Analysis of the outbreak of COVID-19 in Japan on the basis of an SIQR model, Infectious Disease Modelling. 5, 691 (2020)

[14] T. Odagaki, Exact Properties of SIQR model for COVID-19, 
medRxiv preprint doi: https://doi.org/10.1101/2020.08.18.20177709; this version posted November 7, 2020. The copyright holder for this preprint (which was not certified by peer review) is the author/funder, who has granted medRxiv a license to display the preprint in It is made available under a CC-BY-NC-ND 4.0 International license .

https://arxiv.org/abs/2007.12846

[15] L. Young et al., Consequences of delays and imperfect implementation of isolation in epidemic control, Sci Rep 9, 3505 (2019), doi: https://doi.org/10.1038/s41598-019-39714-0Sci

[16] C.P. Vyasarayani and A. Chatterjee, New approximations, and policy implications, from a delayed dynamic model of a fast pandemic, doi: https://doi.org/10.1101/2020.04.09.20059436

[17] M. Utamura et al., Isolation Considered Epidemiological Model for the Prediction of COVID-19 Trend in Tokyo, Japan, doi: https://doi.org/10.1101/2020.07.31.20165829

[18] Japanese COVID-19 data complied by J.A.G Japan corporation: https://gis.jag-japan.com/covid19jp/

[19] J. Bullard et al., Predicting Infectious Severe Acute Respiratory Syndrome Coronavirus 2 From Diagnostic Samples,

Clinical Infectious Diseases ${ }^{\circledR} 2020 ; X X(X X): 1-4$, published online May 22, 2020. DOI: $10.1093 / \mathrm{cid} / \mathrm{ciaa} 638$

[20] R. Woelfel et al., Virological assessment of hospitalized patients with COVID-2019, Nature 581, 465-469(2020)

[21] S. A. Lauer et al., The Incubation Period of Coronavirus Disease 2019 (COVID-19) From Publicly Reported Confirmed Cases: Estimation and Application, Ann. Intern. Med. 172(9), 577-582(2020)

[22] P-Y. Tao et al., Determination of risk factors for predicting the onset of symptoms in asymptomatic COVID-19 infected patients, Int. J. Med. Sci. 2020 (16 Aug), Vol. 17, pp.2187

[23] Y-H. Lee et al., Clinical Course of Asymptomatic and Mildly Symptomatic Patients with Coronavirus Disease Admitted to Community Treatment Centers, South Korea, Emerg. Infect. Dis. 2020 10; 26(10):2346-2352

[24] K. Mizumoto et al., Estimating the asymptomatic proportion of coronavirus diseases 2019 (COVID-19) cases on board the Diamond Princess cruise ship, Yokohama, Japan, 2020, https://www.eurosurveillance.org/content/10.2807/15607917.ES.2020.25.10.2000180 